\title{
Time-Domain Microwave Breast Screening System: Testing with Advanced Realistic Breast Phantoms
}

\author{
Emily Porter, Adam Santorelli, Dady Coulibaly, Mark Coates and Milica Popović \\ Department of Electrical and Computer Engineering \\ McGill University \\ Montreal, Canada \\ \{emily.porter, adam.santorelli, dady.coulibaly\}@mail.mcgill.ca, \{mark.coates, milica.popovich\}@mcgill.ca
}

\begin{abstract}
In this work, we present advanced testing scenarios of our microwave time-domain breast cancer detection system using irregular-shaped breast phantoms. We show how to construct phantoms with uneven skin thickness, asymmetrical breast surface and high amounts of glandular tissue. The results show that our system can detect tumors in certain test scenarios, and we highlight the system configuration that produces the most readily discernible tumor response signals.
\end{abstract}

Keywords-cancer detection; microwave imaging; microwave propagation; phantoms

\section{INTRODUCTION AND MOTIVATION}

Microwave detection methods have been proposed in the literature as a complementary technology to x-ray mammography [1], [2]. While mammography is the 'gold standard' [3] of breast cancer detection, it has been shown to have poor sensitivity and specificity [3]. Based on the inherent contrast in healthy and malignant tissues' dielectric properties, microwave imaging does not require the ionizing radiation of mammography, is non-invasive and pain-free [3].

Our work uses a time-domain microwave system to detect cancerous growths within the breast. Advanced systems in the literature have focused on frequency-domain measurements. We use a time-domain method because: a) the equipment needed is more cost-effective and b) a breast scan can be completed in a very short period of time. The system is described in detail in [4], along with basic test results. In [5], we verified our system's detection abilities on heterogeneous hemi-spherical breast phantoms.

Here, we take our system tests to a higher level - we now use breast phantoms that are more realistic in three ways: the breast phantoms are irregularly-shaped (no longer hemispherical), the skin thickness is variable around the breast, and the glandular structures are asymmetric about the nipple. All of these details can potentially make tumor detection more challenging. The goal of this set of tests is to ensure that our system is able to detect tumors in very complicated phantoms in advance of clinical trials, so that any necessary system adjustments can be made beforehand. This approach can save time and money in the design of a clinically appropriate system.

\section{PREPARING THE BREAST PHANTOMS}

\section{A. Tissue Phantoms}

Skin-, fat-, tumor- and gland-mimicking tissue phantoms have been made from common chemicals. Each tissue phantom has been designed to have dielectric properties matched to those of actual tissue measurements reported in [6]. Tissue concentrations, mixing procedures and electrical properties can be found in [7].

\section{B. Complete Breast Phantom}

In constructing a complete breast phantom, we use two breast-shaped moulds. The breast moulds are identical and measure approximately $17.5 \times 16.5 \mathrm{~cm}^{2}$, with a depth of $8 \mathrm{~cm}$. To start, we compress the skin mixture between the two breastmoulds. The thickness of the skin is controlled by weighing down the top mould with water until the moulds are at the desired separation; we aim for a skin-wide average of $2 \mathrm{~mm}$. On the same day, a glandular mixture is made and allowed to solidify in multiple conical moulds (the cones have an average volume of $28 \mathrm{~mL}$ ). We allow the skin and glands to harden for one day. (They should not be allowed to harden for too long, because the gland phantom is easier to place in the breast when it is still soft.)

At this point, the glandular structures are placed within the skin in the desired number and arrangement. (Note: we place the glands such that the tips of the cones are inside the nipplearea.) The remaining volume interior to the skin is filled with fat-mimicking tissue. We also make tumor phantom in a separate container, from which we manually carve the tumors into the chosen shapes and sizes. Two photographs of a completed breast phantom are shown in Fig. 1.
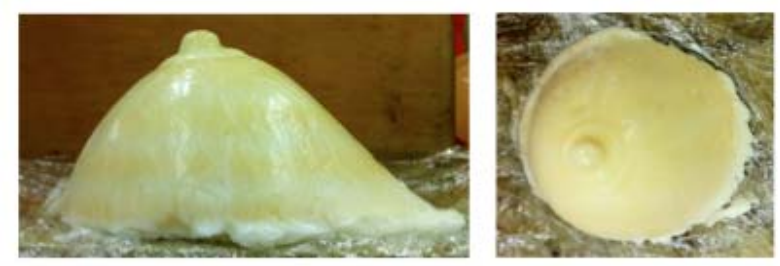

Figure 1. Photographs of a completed breast phantom.

This work was supported by the Natural Sciences and Engineering Research Council of Canada (NSERC), le Fonds québécois de la recherche sur la nature et les technologies (FQRNT) and le Partenariat de Recherche Orientée en Microélectronique, Photonique et Télécommunications (PROMPT). 
For this set of system tests, we make three complete breast phantoms. They all have high glandular content, as this is the most challenging environment for successful tumor detection. In particular, we make phantoms that are $60 \%, 70 \%$ and $80 \%$ glandular tissue, by volume.

\section{MEASUREMENT SET-UP}

We described our microwave system thoroughly in [4]. Here, we provide a very brief overview: an impulse generator transmits a pulse of 70 ps duration to an ultrawideband antenna. This antenna is held in a slot on the exterior of a hemi-spherical bowl-shaped radome, which holds the phantom under test on its inner surface. A fat-mimicking matching medium is used between the radome and the skin to avoid air gaps. A receiving antenna placed elsewhere in the radome picks up the signal that is scattered off of the breast phantom.

The ultrawideband antenna used in our system is the Traveling Wave Tapered and Loaded Transmission Line Antenna (TWTLTLA), described in [8]. This antenna is planar, with appropriately compact dimensions of $0.635 \times 12 \times 15.8 \mathrm{~mm}^{3}$. It is designed for optimized performance in a medium of relative permittivity, $\varepsilon_{r}$, equal to 10.2 ; a value which matches that of the antenna substrate and is also very close to that of healthy adipose tissue within the breast. The TWTLTLA has been shown to have a radiation efficiency of $39.2 \%$ and signal fidelity of 0.95 or better.

Table I lists the relationship between the configuration of the transmitting and receiving antenna for each test case (cases are numbered to be consistent with past work). For example, case 1 indicates a scenario in which the antennas are on opposite sides of the breast and are oriented in the same way (co-polarized). On the other hand, case 7 is a scenario in which the antennas are on the same side of the breast and are oriented perpendicularly to each other (cross-polarized). Cases 5 and 6 appear the same in Table I, the difference between them is that the transmitting and receiving antennas have swapped slots in the radome.

We performed measurements with each of these antenna arrangements on each complete breast phantom. For each phantom, we use three tumors: $0.5 \mathrm{~cm}, 1 \mathrm{~cm}$, and $2 \mathrm{~cm}$ diameter spheres. In cases 1 and 2, the tumor is placed directly in the center of the breast, while in cases 5-7 the tumor is positioned halfway between the center and the radome wall where the antennas are located. The tumor depth is approximately $1 \mathrm{~cm}$ from the chest wall in each case.

\section{MEASUREMENT RESUlts}

In this section, we present a series of measurement results for each scenario described in Table I. The metric we are concerned with is the 'peak tumor response'. We calculate this as the $\mathrm{dB}$ ratio of the maximum of the absolute value of the tumor response to the maximum of the absolute value of the input pulse, where the tumor response is the difference between the healthy baseline and the received signal with tumor present. If we cannot detect the peak tumor response above the noise level then tumor detection is unsuccessful.

Table II provides the peak tumor responses (in $\mathrm{dB}$ relative to the input) for cases 5 and 7 . The values are shown for the
TABLE I. LIST OF MEASUREMENT SCENARIOS

\begin{tabular}{|c|c|c|c|}
\hline Case \# & Polarization & Scenario & Tumor Location \\
\hline 1 & Co- & Transmission & Central \\
\hline 2 & Cross- & Transmission & Central \\
\hline 5 & Co- & Reflection & Non-central \\
\hline 6 & Co- & Reflection & Non-central \\
\hline 7 & Cross- & Reflection & Non-central \\
\hline
\end{tabular}

$60 \%, 70 \%$, and $80 \%$ glandular-content breast phantoms, with all three tumor sizes. An ' $\mathrm{X}$ ' in a table cell indicates that this particular tumor response was not detectable above the noise level, i.e., that detection has failed.

Similarly, Table III lists the measured peak tumor responses for cases 1 and 6 with all considered phantoms and tumors. We note that case 2 does not show up in either Table II or III. The antenna configuration described by case 2, when applied to our system, failed to provide a peak tumor response above the noise level for each and every phantom type and tumor size. Thus we confirm here, as was concluded in [5], that a cross-polarized transmission arrangement of antennas, as in case 2 , is extremely undesirable for tumor detection.

From Table II, we can see that with case 5, a co-polarized reflection scenario, our system detects all three sizes of tumors in all three glandular phantoms. With case 7 only $1 / 9^{\text {th }}$ of tumors are detected. In Table III, we see two cases for which our system obtains intermediate levels of tumor detection. For case 1 , only $1 / 3^{\text {rd }}$ of peak tumor responses were detectable. On the other hand, case 6 enabled detection in all but one of the subcases.

We define the noise level of our experimental system as the threshold below which tumor response signals are undetectable. This threshold is defined by not only the inherent noise in the measurement equipment (which has a noise floor around $-70 \mathrm{~dB}$ ), but also reflection signals not due to the tumor's presence. In other words, multiple reflections off of tissues within a breast phantom (skin, gland) lead to received signals that are noisy due to both measurement noise and these undesirable reflections. The noise level is not constant over phantom types and cases; the number of glands and gland positioning relative to antennas will affect the amount of undesirable reflections. For example, the $70 \%$ gland phantom has eight subcases for which the tumor was undetectable, marked by ' $\mathrm{X}$ ' in Tables II and III. These undetectable peak tumor responses, occurring in cases 1,2 and 7 , range in size from $-64.3 \mathrm{~dB}$ to $-60.1 \mathrm{~dB}$. However, the peak tumor response of $-61.6 \mathrm{~dB}$ is detectable in case 5 (small tumor). Thus there is no single noise threshold for our system, but instead the level at which a tumor response can be detected depends entirely on the setting (phantom tissue types, antenna positioning relative to each other, antenna locations with respect to tissues).

TABLE II. MEASUREMENT RESUltS FROM CASES 5 AND 7: PEAK TUMOR RESPONSES (IN DB RELATIVE TO INPUT PULSE) FOR $60 \%, 70 \%$ AND $80 \%$ GLAND PHANTOMS WITH THREE TUMOR SizES

\begin{tabular}{|c|c|c|c|c|c|c|}
\cline { 2 - 7 } \multicolumn{1}{c|}{} & \multicolumn{3}{c|}{ CASE 5 } & \multicolumn{3}{c|}{ CASE 7 } \\
\cline { 2 - 7 } \multicolumn{1}{c|}{} & $\mathbf{0 . 5} \mathbf{c m}$ & $\mathbf{1} \mathbf{c m}$ & $\mathbf{2} \mathbf{~ c m}$ & $\mathbf{0 . 5} \mathbf{c m}$ & $\mathbf{1} \mathbf{c m}$ & $\mathbf{2} \mathbf{c m}$ \\
\hline $\mathbf{6 0 \%}$ gland & -56.1 & -52.5 & -51.0 & $\mathrm{X}$ & $\mathrm{X}$ & $\mathrm{X}$ \\
\hline $\mathbf{7 0 \%}$ gland & -61.6 & -58.1 & -52.1 & $\mathrm{X}$ & $\mathrm{X}$ & -57.6 \\
\hline $\mathbf{8 0 \%}$ gland & -52.6 & -52.3 & -53.7 & $\mathrm{X}$ & $\mathrm{X}$ & $\mathrm{X}$ \\
\hline
\end{tabular}


TABLE III. MEASUREMENT RESUlTS FROM CASES 1 AND 6: PEAK TUMOR RESPONSES (IN DB RELATIVE TO INPUT PULSE) FOR 60\%, 70\%, AND $80 \%$ Gland PHANTOMS WiTH THREE TuMOR Sizes

\begin{tabular}{|c|c|c|c|c|c|c|}
\cline { 2 - 7 } \multicolumn{1}{c|}{} & \multicolumn{3}{c|}{ CASE 1 } & \multicolumn{3}{c|}{ CASE 6 } \\
\cline { 2 - 8 } \multicolumn{1}{c|}{} & $\mathbf{0 . 5} \mathbf{c m}$ & $\mathbf{1} \mathbf{c m}$ & $\mathbf{2} \mathbf{~ m}$ & $\mathbf{0 . 5} \mathbf{c m}$ & $\mathbf{1} \mathbf{c m}$ & $\mathbf{2} \mathbf{c m}$ \\
\hline $\mathbf{6 0 \%}$ gland & $\mathrm{X}$ & $\mathrm{X}$ & $\mathrm{X}$ & $\mathrm{X}$ & -57.4 & -57.6 \\
\hline $\mathbf{7 0 \%}$ gland & $\mathrm{X}$ & $\mathrm{X}$ & $\mathrm{X}$ & -57.5 & -57.6 & -56.5 \\
\hline $\mathbf{8 0 \%}$ gland & -56.6 & -53.8 & -50.3 & -58.3 & -57.0 & -56.7 \\
\hline
\end{tabular}

Case 5 provides an average peak tumor response (over the three phantom types) of $-56.8 \mathrm{~dB}$ for small tumors, $-54.3 \mathrm{~dB}$ for medium tumors and $-52.3 \mathrm{~dB}$ for large tumors. These values might suggest that increasing tumor sizes leads to increasing peak tumor responses. Yet if we examine the results for the $80 \%$ glandular content phantom with case 5 , we see that the larger tumor in fact has a smaller peak tumor response than do the medium and small tumors. This effect is not unexpected, and has been noted previously in [5]. Due to the complex and highly heterogeneous interior of the breast phantoms, the tumor responses are extremely difficult to predict and are greatly affected by gland position and arrangement within the breast, relative to both the antenna and tumor locations.

In previous work on hemi-spherical glandular phantoms, cases 5, 6 and 7 were all shown to enable detection of various sizes of tumors in $30 \%, 50 \%$ and $80 \%$ glandular phantoms [5]. Here, however, the system with antennas arranged as per case 5 or 6 far outperforms the system with the case 7 antenna arrangement. This shows that the irregular breast shape and skin thickness do in fact increase the detection challenge, and demonstrates the importance of testing the system with more complicated models.

In particular, the large $2-\mathrm{cm}$ tumor in the $70 \%$ gland phantom measured with case 7 leads to a lower peak tumor response than does the same subcase measured with either both cases 5 and 6 . This indicator, along with the failure of case 7 to enable detection in eight of the scenarios, suggests the crosspolarized scattered tumor response is not as useful as other arrangements when the breast is irregular-shaped and heterogeneous.

Comparing all of the cases, it is clear that case 5 leads to the best system performance. Case 5 is the only one for which all three peak tumor responses were clearly seen in each phantom type. Case 6, with its near-perfect detection record, is the second most beneficial case for tumor detection. Since cases 5 and 6 both have co-polarized antennas arranged in a reflection scenario, it is likely that for our system, in particular, this is the best type of antenna layout for tumor detection.

\section{SAFTEY}

In advance of clinical trials, it is key that the safety of our system can be ensured. In the microwave range, the main concern for safety is the heating of tissues due to energy absorption from the incident wave [9]. The specific absorption rate (SAR) characterizes this phenomenon, and is usually the parameter considered to assess safety standards of devices.

For our system, we use the guidelines for safety limits derived from Health Canada's Safety Code 6. The limits in the
United States and European Union are similar. In particular, our system must adhere to the general SAR limits, as well as those of peak field strengths for pulsed fields. The regulations necessitate a peak spatial SAR (averaged over $1 \mathrm{~g}$ of tissue) of $8 \mathrm{~W} / \mathrm{kg}$ for the head, neck and trunk in a controlled scenario, and $1.6 \mathrm{~W} / \mathrm{kg}$ in an uncontrolled environment. Since participants in a trial or patients undergoing tests would be aware of their exposure to fields and associated risks, our system falls in to the controlled environment category. However, we wish to meet the safety requirements for an uncontrolled environment as well, so that the detection system will be more easily approved.

We calculate the maximum power transmitted from one of our antennas using extremely conservative measures: we ignore cable losses and mismatch losses; and assume an antenna efficiency of $50 \%$ (in simulations of the antenna, it was shown to have an efficiency of only $39 \%$ [8]). This procedure ensures that the values we calculate will be higher than the actual values experienced during a breast scan, so if these values fall within safe limits than the real values are guaranteed to. We also note that for pulsed fields, in the frequency range from 1.5 - $15 \mathrm{GHz}$, a 6 min averaging time is used in determining exposure limits [9].

Applying the assumptions in the above paragraph, and knowing the signal level that is fed into the antenna, we are able to obtain the power radiated from one of our antennas. The calculated peak power that is emitted is approximately $0.4 \mathrm{~W}$. Our system currently allows for up to 16 antennas, each of which will transmit to and receive from all other antennas. This results in $16 * 15=240$ pulses that are required to achieve a complete data set. Also, recall that the pulse duration is $70 \mathrm{ps}$.

Using the above values, we apply the following formula, provided in [9]:

$$
W=\frac{1}{6} \sum_{i=1}^{n} W_{i} \Delta t_{i}
$$

where $\mathrm{W}$ is the time-averaged power density, $\mathrm{W}_{\mathrm{i}}$ is the sampled power density in the $\mathrm{i}^{\text {th }}$ time period, $\Delta t_{i}$ is the time duration in minutes of the $i^{\text {th }}$ time period, and $n$ is the number of time periods within $6 \mathrm{~min}$. The relation

$$
\sum_{i=1}^{n} \Delta t_{i}=6 \mathrm{~min}
$$

must also be satisfied. Without measurement, we cannot be sure of the power density at various locations in the breast. Since we do, however, know the power, we can use this in place of power density on both sides of (1). The following parameter values are used in (1) and (2): $n=240, W_{i}=0.4 \mathrm{~W}$ and $\Delta t_{i}=70 p s * \frac{1 \mathrm{~min}}{60 \mathrm{~s}}=1.17 \times 10^{-12} \mathrm{~min}$. We note that when no pulse is being sent, the $W_{i}$ value is equal to zero, thus making the corresponding product with $\Delta t_{i}$ equal to zero as well. For this reason, we only need to perform the sum for the time durations in which the pulse is active. The result is a time-averaged power density for the 240 required pulses of $1.87 \times 10^{-11} \mathrm{~W}$. Thus the peak SAR in $1 \mathrm{~g}$ of tissue would be $1.87 \times 10^{-8} \mathrm{~W} / \mathrm{kg}$.

Alternatively, if we assume that pulses are transmitted continuously over the entire $6 \mathrm{~min}$ period, we can obtain the 
peak SAR for the system regardless of the number of antennas used. In this case, we have a $25 \mathrm{MHz}$ pulse repetition rate, which is active for the whole 6 minutes. This frequency of repetition corresponds to 1 pulse every $40 \mathrm{~ns}$. Therefore, in $6 \mathrm{~min}(360 \mathrm{~s})$, there is a total of $n=9 \times 10^{9}$ pulses transmitted. Using this $n$ value in (1), we calculate $W=7 \times 10^{-4} \mathrm{~W}$. This leads to a time-averaged SAR over $1 \mathrm{~g}$ of tissue of $0.7 \mathrm{~W} / \mathrm{kg}$.

Regardless of whether 240 or $9 \times 10^{9}$ pulses are transmitted in the $6 \mathrm{~min}$ period, it is clear that the resulting SAR values are well below the recommended safety limits. The worst case, with the larger number of pulses transmitted, leads to an SAR of $0.7 \mathrm{~W} / \mathrm{kg}$, which is less than both the uncontrolled environment limit of $1.6 \mathrm{~W} / \mathrm{kg}$ and the controlled limit $8 \mathrm{~W} / \mathrm{kg}$.

There is also a limit of $100 \mathrm{kV} / \mathrm{m}$ on the peak instantaneous electrical field strength [9]. This is an extremely high value and our system does not have sufficient input power to generate such large fields. Thus, we can be certain that the system does not exceed this limit.

While this section has shown only rough estimates, and exact measurements should be completed before testing on human patients commences, the above discussion shows that the system is likely safe for human interaction. It also implies that there may be a bit of room to increase the power to each pulse (in order to increase the resulting tumor response level), particularly if the pulse repetition rate is decreased or if we only transmit exactly the number of pulses that we require (240).

Lastly, we note that the low frequency content $(<1.5 \mathrm{GHz})$ generated by our off-the-shelf impulse generator have not been included in this safety assessment. These frequencies need to be filtered out before the pulse is fed into the transmitting antenna or, a similar analysis as above could be done to ensure safety. This is necessary since safety requirements become more stringent at lower frequencies [9]; for instance, for an uncontrolled environment, the maximum power density at $200 \mathrm{MHz}$ is $2 \mathrm{~W} / \mathrm{m}^{2}$ whereas at $2 \mathrm{GHz}$ the allowable limit is $10 \mathrm{~W} / \mathrm{m}^{2}$. As we do not intend to continue operating our system with such low frequency content, we are not concerned with this issue.

\section{CONCLUSION}

In this paper, we have presented a procedure for fabricating breast phantoms with irregular shape, similar to that of an actual breast. We further used these phantoms to test the behaviour of our microwave time-domain breast cancer detection system. The measurement results confirm previous work that suggested co-polarized antennas recording a signal reflected off of the breast as the most optimal antenna layout for tumor detection. The data from this set of tests did not match well with measurements previously performed on hemi-spherical shaped breast phantoms; indicating that the irregular breast and skin shape are indeed important factors that have a significant effect on the resulting signals. Finally, we presented calculations that demonstrate the safety of our detection system.

\section{ACKNOWLEDGMENT}

We would like to thank Jules Gauthier and his colleagues at Polytechnique Montreal for fabricating the antennas for us. We are also grateful to Jean-Marc Gauthier and Badawy Sha'ath, in the McGill University Chemistry Department, for letting us use their chemistry lab to make the breast phantoms and store all of our chemicals. Finally, we thank McGill University undergraduate student Alexandre Bourdon for his design of the gland-shaped cones.

\section{REFERENCES}

[1] M. Klemm, D. Gibbins, J. Leendertz, T. Horseman, A. W. Preece, R. Benjamin and I. J. Craddock, "Development and testing of a 60-Element UWB conformal array for breast cancer imaging," in Proc. 5th European Conference on Antennas and Propagation (EUCAP 2011), Rome, Italy, April 11-15, 2011.

[2] Younju Lee, Hyuk Je Kim, Jong Moon Lee, Soon Ik Jeon, Tomasz M. Gregorczyk, and P.M. Meaney, "Microwave tomography technology test-bed system for study breast cancer detection technology based on electromagnetic field," in Proc. IEEE Antennas and Propagation Society International Symposium, 2008 (AP-S 2008), San Diego, California, July 5-11, 2008.

[3] E.C. Fear, P.M. Meaney and M.A. Stuchly "Microwaves for breast cancer detection?" IEEE Potentials, pp.12-18, February/March 2003.

[4] E. Porter, A. Santorelli, M. Coates and M. Popović, "An experimental system for time-domain microwave breast imaging," in Proc. 5th European Conference on Antennas and Propagation (EUCAP 2011), Rome, Italy, April 11-15, 2011.

[5] E. Porter, A. Santorelli, A. Bourdon, D. Coulibaly, M. Coates and M. Popović, "Time-domain microwave breast cancer detection: experiments with comprehensive glandular phantoms," in Proc. 2011 Asia-Pacific Microwave Conference (APMC 2011), Melbourne, Australia, Dec. 5-8, 2011.

[6] M. Lazebnik, D. Popovic, L. McCartney, C. Watkins, M, Lindstrom, J. Harter, S. Sewall, T. Ogilvie, A. Magliocco, T. Breslin, W. Temple, D. Mew, J. Booske, M. Okoniewski and S. Hagness, "A large-scale study of the ultrawideband microwave dielectric properties of normal, benign and malignant breast tissues obtained from cancer surgeries," Phys. Med. Biol., Vol. 52, pp. 6093-6115, 2007.

[7] E. Porter, J. Fakhoury, R. Oprisor, M. Coates and M. Popović, "Improved tissue phantoms for experimental validation of microwave breast cancer detection," in Proc. 4th European Conference on Antennas and Propagation (EUCAP 2010), Barcelona, Spain, April 12-16, 2010.

[8] H. Kanj and M. Popović, "A Novel Ultra-Compact Broadband Antenna for Microwave Breast Tumor Detection," Progress in Electromagnetics Research, vol. 86, pp. 169-198, 2008.

[9] Health Canada, "Limits of Human Exposure to Radiofrequency Electromagnetic Energy in the Frequency Range from $3 \mathrm{kHz}$ to 300 GHz: Safety Code 6," Health Canada, 2009. 